\title{
The Sum of Like Powers of the Zeros of the Riemann Zeta Function
}

\author{
By D. H. Lehmer
}

\begin{abstract}
In this paper we discuss a method of evaluating the sum $\sigma_{r}=\sum \rho^{-r}$ where $r$ is an integer greater than 1 and the sum is taken over all the complex zeros of $s(s)$, the Riemann zeta function. The method requires the coefficients of the Maclaurin expansion of the entire function $f(s)=(s-1) \zeta(s)$. These are obtained from a limit theorem of Sitaramachandrarao by the use of the Euler-Maclaurin summation formula. The sum $\sigma_{r}$ is then obtained from the logarithmic derivative of the function $f(s)$. A table of $\sigma_{r}$ is given to 30 decimals for $r=2(1) 26$.
\end{abstract}

Despite the vast literature and machine computing on the zeros of

$$
\varsigma(s)=\sum_{n=1}^{\infty} n^{-s},
$$

no one has attempted to evaluate

$$
\sigma_{2}=\sum_{\rho} \rho^{-2}
$$

the sum extending over all the complex zeros $\rho$ of $\varsigma(s)$.

The direct approach, summing over the known zeros, is not effective. For example, we find the first 50 zeros sum to

$$
\sum_{|\rho| \leq 88.8} \rho^{-2}=-.034721
$$

whereas the infinite series (1) is nearly a time and a half as much, as we shall see. The Dirichlet series representation of functions of a complex variable is not conducive to the solution of this problem, which needs a power series approach.

In a letter to Hermite [1], Stieltjes gave the expression

$$
\zeta(s)=\frac{1}{s-1}+\sum_{n=0}^{\infty} \frac{(-1)^{n} \gamma_{n}}{n !}(s-1)^{n},
$$

where $\gamma_{n}$ is a generalization of Euler's constant $\gamma$,

$$
\gamma_{n}=\lim _{N \rightarrow \infty}\left\{\sum_{k=1}^{N} \frac{(\log k)^{n}}{k}-\frac{(\log N)^{n+1}}{n+1}\right\} .
$$

Briggs and Chowla [2] give two proofs of this result. Liang and Todd [3] call them Stieltjes constants and give a table of $\gamma_{n}$ for $n \leq 19$ to 15 significant decimals, improving the earlier results by Jensen [4] and Gram [5].

Received November 3, 1986; revised April 13, 1987.

1980 Mathematics Subject Classification (1985 Revision). Primary 11M26. 
The power series in (2) is an entire function of $s$ and hence has an infinite radius of convergence.

To find, more generally,

$$
\sigma_{k}=\sum_{\rho} \rho^{-k}
$$

we need an expansion in powers of $s$, not $(s-1)$. In executing the transformation so much accuracy is lost that it was decided to abandon the method. What we need is a method of computing $\varsigma^{(k)}(0)$. It is well known that

$$
\varsigma(0)=-\frac{1}{2}, \quad \varsigma^{\prime}(0)=-\frac{1}{2} \log 2 \pi .
$$

Ramanujan [6, p. 25] gave

$$
\varsigma^{\prime \prime}(0)=-\frac{1}{2}(\log 2 \pi)^{2}+\frac{\pi^{2}}{24}-a_{1},
$$

and Apostol [7] gives a formula for $\varsigma^{(k)}(0)$ and a table for $k \leq 18$ to $15 \mathrm{D}$ in terms of $a_{n}$ defined by

$$
\sum_{n=0}^{\infty} a_{n}(s-1)^{n}=\Gamma(s) \zeta(s)+\frac{1}{1-s} .
$$

We must regard the $a$ 's as unknowns and as difficult to approximate as $\zeta^{(k)}(0)$ itself.

It was decided to use an ab initio approach via a recent theorem of Sitaramachandrarao [8] to the effect that

$$
\varsigma(s)+\frac{1}{1-s}=\sum_{n=0}^{\infty} \frac{(-1)^{n} \delta_{n}}{n !} s^{n},
$$

where

$$
\delta_{n}=\lim _{N \rightarrow \infty}\left\{\sum_{k=1}^{N}(\log k)^{n}-\int_{1}^{N}(\log t)^{n} d t-\frac{1}{2}(\log N)^{n}\right\} .
$$

This is an analogue of Stieltjes' theorem.

To calculate $\delta_{n}$ we use the Euler-Maclaurin summation formula $[9$, p. 806]

$$
\begin{aligned}
\sum_{i=0}^{m} F(a+i)= & \int_{a}^{b} F(t) d t+\frac{1}{2} F(b)+\frac{1}{2} F(a) \\
& +\sum_{k=1}^{p-1} \frac{B_{2 k}}{(2 k) !}\left\{F^{(2 k-1)}(b)-F^{(2 k-1)}(a)\right\}+R_{p}
\end{aligned}
$$

where $m=b-a$ and $B_{2}=\frac{1}{6}, B_{4}=-\frac{1}{30}$ are the Bernoulli numbers. We also use the Stirling numbers of the first kind. These latter are denoted by $s(i, j)$ and are generated by $[9$, p. 824]

$$
\sum_{j=0}^{i} s(i, j) x^{j}=x(x-1)(x-2) \cdots(x-i+1)
$$

and enjoy the recurrence

$$
s(i+1, j)=s(i, j-1)-i s(i, j) \quad(s(0,0)=1) .
$$


We have the following lemma:

LEMMA. If $h$ is a nonnegative integer, then

$$
\frac{d^{h}}{d x^{h}}\left\{(\log x)^{n}\right\}=x^{-h} n ! \sum_{k=0}^{n} \frac{(\log x)^{k}}{k !} s(h, n-k) .
$$

Proof. The proof is by induction on $h$. The lemma is easily seen to be true for $h=0$, since $s(0, j)=0$, except for $s(0,0)=1$. If true for $h$, we have

$$
\begin{aligned}
\frac{d^{h+1}}{d x^{h+1}}\left\{(\log x)^{n}\right\}= & -h x^{-(h+1)} n ! \sum_{k=0}^{n} \frac{(\log x)^{k}}{k !} s(h, n-k) \\
& +x^{-(h+1)} n ! \sum_{k=1}^{n} \frac{(\log x)^{k-1}}{(k-1) !} s(h, n-k) \\
= & x^{-(h+1)} n ! \sum_{k=0}^{n} \frac{(\log x)^{k}}{k !}(s(h, n-k-1)-h s(h, n-k)) \\
= & x^{-(h+1)} n ! \sum_{k=0}^{n} \frac{(\log k)^{k}}{k !} s(h+1, n-k) .
\end{aligned}
$$

Thus the induction is complete.

Before we apply (6) we fix a number $\nu \leq N$ and write

$$
\sum_{k=1}^{N}(\log k)^{n}=\sum_{k=1}^{\nu-1}(\log k)^{n}+\sum_{k=\nu}^{N}(\log k)^{n}=S_{1}+S_{2} .
$$

We plan to evaluate $S_{1}$ on our machine. As to $S_{2}$, we apply (6) with $m=N-\nu$, $b=N, a=\nu$ and $F(t)=(\log t)^{n}$ to get from the lemma

$$
\begin{aligned}
S_{2}= & \int_{\nu}^{N}(\log t)^{n} d t+\frac{1}{2}(\log N)^{n}+\frac{1}{2}(\log \nu)^{n} \\
& +\sum_{k=1}^{p-1} \frac{B_{2 k}}{(2 k) !} \sum_{j=0}^{n-1} \frac{n !}{j !} s(2 k-1, n-j)(\log N)^{j} N^{1-2 k} \\
& -\sum_{k=1}^{p-1} \frac{B_{2 k}}{(2 k) !} \sum_{j=0}^{n-1} \frac{n !}{j !} s(2 k-1, n-j)(\log \nu)^{j} \nu^{1-2 k}+R_{p} .
\end{aligned}
$$

Substituting this into (5), cancelling and letting $N \rightarrow \infty$ we find

$$
\begin{aligned}
\delta_{n}= & \sum_{k=1}^{\nu}(\log k)^{n}-\int_{1}^{\nu}(\log t)^{n} d t-\frac{1}{2}(\log \nu)^{n} \\
& -\sum_{k=1}^{p-1} \frac{B_{2 k}}{(2 k) ! \nu^{2 k-1}} \sum_{j=0}^{n-1} \frac{n !}{j !} s(2 k-1, n-j)(\log \nu)^{j}+R_{p} .
\end{aligned}
$$




\section{TABLE 1}

\begin{tabular}{|c|c|}
\hline$n$ & $\delta_{n}$ \\
\hline 0 & .500000000000000000000000000000 \\
\hline 1 & -.081061466795327258219670263594 \\
\hline 2 & -.006356455908584851210100026730 \\
\hline 3 & .004711166862254447761060813366 \\
\hline 4 & .002896811986292041012780472259 \\
\hline 5 & .000232907558454724535985837796 \\
\hline 6 & -.000936825130050929504283508545 \\
\hline 7 & -.000849823765001669151706027602 \\
\hline 8 & -.000232431735511559582855690064 \\
\hline 9 & .000330589663612296445256127250 \\
\hline 10 & .000543234115779708472231988943 \\
\hline 11. & .000375493172907263650467030884 \\
\hline 12 & - .00001960 3536281013919766484025 \\
\hline 13 & -.000407241232563033143432121367 \\
\hline 14 & -.0005 70492013281777715641291384 \\
\hline 15 & -.00039392 7078981204421827660819 \\
\hline 16 & .000083458805825501681727648805 \\
\hline 17 & .000660943729628596896169402998 \\
\hline 18 & .001026227286540854002177014155 \\
\hline 19 & .000865575776779282991576072414 \\
\hline 20 & .000019293671783705140106329976 \\
\hline 21 & -.001356906052134549461149137833 \\
\hline 22 & -.002692156458753291284034257109 \\
\hline 23 & -.003051385621241627138845437386 \\
\hline 24 & -.001424291849418545853222186792 \\
\hline 25 & .002707789212886006788197482192 \\
\hline 26 & .008602880969279324256140452025 \\
\hline 27 & .013556162030983539621697371623 \\
\hline 28 & .012778513326691412737021780989 \\
\hline 29 & .000576026175993012089937446958 \\
\hline 3 & -.026465704147079752693730404860 \\
\hline
\end{tabular}

This expression for $\delta_{n}$ depends on the two parameters $\nu$ and $p$. The optimal choice of the parameters depends on $n$ but this dependence complicates the programming. There is also a dependence on the desired accuracy of $\delta_{n}$ and accordingly we must use sufficiently precise logarithms. This is no simple matter. In experimenting with (8), $\left|\delta_{n}\right|$ grows very slowly, but the four terms of (8) are decidedly unbounded. In particular, therefore, the sum of the first three terms is nearly the negative of the fourth term. For example if $\nu=180, p=15$ and $n=24$, the first three terms contribute

$$
316442088365362.25420772 \ldots
$$

whereas the fourth term is

$$
-316442088365362.25563201 \ldots \text {. }
$$

This gives

$$
\delta_{24}=-.00142429 \text {. }
$$

Only six significant decimals remain from the destructive cancellation of numbers of 23 significant decimals. This loss of significance increases with $n$, but is relatively harmless for $n \leq 6$. 
TABLE 2

\begin{tabular}{|c|c|}
\hline & $c_{n}$ \\
\hline & .5000000000000000000000 \\
\hline & .081061466795327258219670263594 \\
\hline & -.003178227954292425605050013365 \\
\hline & -.000785194477042407960176802228 \\
\hline & .000120700499428835042199186344 \\
\hline & -.000001940896320456037799881982 \\
\hline & -.000001301146013959624311504873 \\
\hline & .000000168615826389220069782942 \\
\hline & -.000000005764675979949394416064 \\
\hline & -.000000000911016489231416570922 \\
\hline & .000000000149700759419011373521 \\
\hline & -.000000000009406895665666176910 \\
\hline & -.000000000000040925826304158315 \\
\hline & .000000000000065399048058910152 \\
\hline & -.000000000000006543968749891919 \\
\hline & .000000000000000301242487136679 \\
\hline & .000000000000000003988894706311 \\
\hline & -.000000000000000001858215043379 \\
\hline & .000000000000000000160288563853 \\
\hline & -.000000000000000000007115582739 \\
\hline & .000000000000000000000007930312 \\
\hline & .000000000000000000000026558642 \\
\hline & -.000000000000000000000002395155 \\
\hline & .000000000000000000000000118033 \\
\hline & -.000000000000000000000000002296 \\
\hline & -.000000000000000000000000000175 \\
\hline & .000000000000000000000000000021 \\
\hline & -.00000000000000000000000000000 \\
\hline
\end{tabular}

It was decided to run a program for $\delta_{n}$ that exploited certain recursive features of (8). For example, if

$$
I_{n}=\int_{1}^{\nu}(\log t)^{n} d t
$$

then

$$
I_{n}=\nu(\log \nu)^{n}-n I_{n-1} \quad\left(I_{0}=\nu-1\right) .
$$

It was also decided to compute $\delta_{1}, \delta_{2}, \ldots, \delta_{30}$ with accuracy sufficient to determine $\delta_{n}$ from 44 significant figures for $n=1$ to about 10 for $n=30$. The details of this program with its 1024 instructions and its multiprecision subroutines will not be given. The estimation of the remainder $R_{p}$ was avoided by the following considerations. The denominator factor $\nu^{2 k-1}$ assures us of terms in the asymptotic series that decrease by a factor of at least $10^{4}$ for $\nu>100$. The machine was instructed to choose $p$ so that the last term it computed was less than $10^{-40}$, so that $\left|R_{p}\right|<10^{-40}$. Two runs were made with $\nu=180$ and $\nu=200$. The two results were compared and only the digits that were common to the two runs were retained. Table 1 gives the values of $\delta_{n}$. 


\title{
TABLE 3
}

$n$

0

\begin{abstract}
$d_{n}$
.500000000000000000000000000000 .418938533204672741780329736406 .084239694749619683824720276959

- .0023 93033477250017644873211137

- .000905894976 471243002375988572 .000122641395749291079999068326

$-.000000639750306496413488377109$

- .0000 01469761840348844381287815 .000000174380502369169464199006

- .0000 00004853659490717977845142

- .000000001060717248650427944443 .000000000159107655084677550430

$-.000000000009365969839362018594$

- .0000 00000000106324874363068468 .000000000000071943016808802072

$-.000000000000006845211237028598$ .000000000000000297253592430368 .000000000000000005847109749690

$-.000000000000000002018503607231$ .000000000000000000167404146592

$-.000000000000000000007123513051$

$-.000000000000000000000018628330$ .000000000000000000000028953798

$-.000000000000000000000002513188$ .000000000000000000000000120328

$-.000000000000000000000000002121$

- .000000000000000000000000000196 .000000000000000000000000000023

$-.000000000000000000000000000001$
\end{abstract}

We use the following notation.

(9)

$$
\begin{gathered}
\zeta(s)=\frac{1}{s-1}+\sum_{n=0}^{\infty}(-1)^{n} \delta_{n} s^{n} / n !=\frac{1}{s-1}+\sum_{n=0}^{\infty} c_{n} s^{n}, \\
(s-1) \varsigma(s)=\sum_{n=0}^{\infty} d_{n} s^{n} \quad\left(d_{0}=\frac{1}{2}, d_{n}=c_{n-1}-c_{n}\right), \\
g(s)=2(s-1) \zeta(s), \\
\frac{g^{\prime}(s)}{g(s)}=\sum_{n=0}^{\infty} b_{n} s^{n} \quad\left(b_{i}=2\left[(i+1) d_{i+1}-\sum_{j=0}^{i-1} b_{j} d_{i-j}\right], i=0,1, \ldots\right) .
\end{gathered}
$$

The coefficients $c_{n}, d_{n}$ and $b_{n}$ are given in Tables 2, 3 and 4 .

In terms of the complex zeros of $\varsigma(s)$, we have the Weierstrass expansion $[9, \mathrm{p}$. 807]

$$
\varsigma(s)=\frac{\exp \left\{s\left(\log 2 \pi-1-\frac{1}{2} \gamma\right)\right\}}{2(s-1) \Gamma\left(\frac{1}{2} s+1\right)} \prod_{\rho}\left(1-\frac{s}{\rho}\right) e^{s / \rho} .
$$

Multiplying this by $2(s-1)$ and taking logarithms, we get

(10) $\log g(s)=s\left(\log 2 \pi-1-\frac{1}{2} \gamma\right)-\log \Gamma\left(\frac{1}{2} s+1\right)+\sum_{\rho}\left(\log \left(1-\frac{2}{\rho}\right)+\frac{s}{\rho}\right)$. 
TABLE 4

$\begin{array}{rr}n & b_{n} \\ & \\ 0 & .837877066409345483560659472811 \\ 1 & -.365079199416252006360995801282 \\ 2 & .150368271126401391597729938428 \\ 3 & -.06771882932820782648 \text { 8077 0023 41 } \\ 4 & .032403277254874547590119370358 \\ 5 & -.015895703907090079041777735937 \\ 6 & .007877733303737421789458029521 \\ 7 & -.003922178441516331026458708225 \\ 8 & .001957047613964070274475997797 \\ 9 & -.000977533758775545001810182006 \\ 10 & .000488522553198424083329975007 \\ 11 & -.000244200704753666874030085575 \\ 12 & .000122085292155703530612285356 \\ 13 & -.000061038894539355402858559125 \\ 14 & .000030518511603896796797057213 \\ 15 & -.000015259022251273374837382239 \\ 16 & .000007629452798443861451996350 \\ 17 & -.000003814711827443176944967933 \\ 18 & .000001907352272439416206237585 \\ 19 & -.000000953675226175340545978734 \\ 20 & .000000476837385622495072294187 \\ 21 & -.000000238418635952592546329924 \\ 22 & .000000119209303762903924626477 \\ 23 & -.000000059604648328315559104972 \\ 24 & .000000029802323275908932526451 \\ 25 & -.000000014901161415898126786425 \\ 26 & .000000007450580652435956819620 \\ 27 & -.000000003725290312339864763575 \\ 28 & .000000001862645152700431129919 \\ 29 & -.000000000931322575482844777796 \\ 30 & .000000000465661287524580446306\end{array}$

We note that

$$
\sum_{\rho}\left(\log \left(1-\frac{s}{\rho}\right)+\frac{s}{\rho}\right)=-\sum_{\rho} \sum_{r=2}^{\infty} \frac{s^{r}}{r \rho^{r}}
$$

Differentiating (10) with respect to $s$ and using the formula [9, p. 259, 6.3.14],

$$
\frac{d}{d s} \log \Gamma\left(\frac{s}{2}+1\right)=\frac{1}{2} \psi\left(\frac{s}{2}+1\right)=-\frac{\gamma}{2}+\sum_{n=2}^{\infty}(-1)^{n} \frac{\zeta(n)}{2^{n}} s^{n-1}
$$

we get

$$
\frac{g^{\prime}(s)}{g(s)}=\log 2 \pi-1-\sum_{n=2}^{\infty} \frac{(-1)^{n} \zeta(n)}{2^{n}} s^{n-1}-\sum_{\rho} \sum_{r=2}^{\infty} s^{r-1} \rho^{-r} .
$$

Identifying the coefficient of $s^{m}$ in both (11) and (9), we get

$$
b_{0}=\log 2 \pi-1, \quad b_{m}=\frac{(-1)^{m} \zeta(m+1)}{2^{m+1}}-\sigma_{m+1} \quad(m>0),
$$

where $\sigma_{k}$ is defined in (3). Hence,

$$
\sigma_{r}=-\left(\frac{(-1)^{r} \varsigma(r)}{2^{r}}+b_{r-1}\right), \quad r>1 .
$$


TABLE 5

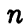

$$
\begin{array}{r}
-.000111158231452105922762668239 \\
.000073627221261689518326771307 \\
.000000715093355762607735801094 \\
-.000000281436416938766261606716 \\
-.000000004574191149704772111163 \\
.000000001268868110950760719013 \\
.000000000028274371550558870893 \\
-.000000000005997714847151874595 \\
-.000000000000169068876025921158 \\
.000000000000028738151300925908 \\
.000000000000000983296676136499 \\
-.000000000000000137926293617300 \\
-.000000000000000005591337659272 \\
.000000000000000000659812483976 \\
.000000000000000000031215867676 \\
-.000000000000000000003138649684 \\
-.000000000000000000000171655589 \\
.000000000000000000000014823993 \\
.000000000000000000000000932009 \\
-.000000000000000000000000069429 \\
-.000000000000000000000000005006 \\
.000000000000000000000000000322 \\
.000000000000000000000000000027 \\
-.000000000000000000000000000001
\end{array}
$$$$
046154317295804602757107990379
$$

In particular,

$$
\sigma_{2}=-\left(\frac{\pi^{2}}{24}+b_{1}\right)=-.046154317295804602757107990379
$$

and

$$
\sigma_{3}=-\left(-\frac{\varsigma(3)}{8}+b_{2}\right)=-.000111158231452105922762668239 .
$$

These two numbers are not connected in any obvious way with any other known constants. Their continued fractions show no radical departure from the norm.

Formula (12) may be used to compute other values of $\sigma_{r}$. The term $\varsigma(r) / 2^{r}$ represents the sum of the negative $r$ th powers of the trivial zeros $-2 n(n=1,2,3, \ldots)$ of $\varsigma(s)$. Values of $\zeta(n)$ were taken from the 50D tables of Lienard [10]. The values of $\sigma_{n}$ are given in Table 5 .

Table 5 can be used to evaluate such sums as

$$
\sum_{\rho} \frac{1}{\rho(\rho-1)}=\sum_{r=2}^{\infty} \sigma_{r}=-.046191417932242067628620495813
$$

and

$$
\sum_{\rho} \frac{1}{\rho(\rho+1)}=\sum_{r=2}^{\infty}(-1)^{r} \sigma_{r}=-.045970522563879642410855671356 .
$$

These sums are special cases of

$$
\sum_{\rho} \frac{1}{\rho(\rho-a)}=\sum_{r=2}^{\infty} \sigma_{r} a^{r-2}
$$


The author owes a debt of gratitude to the referee who went to the trouble of reproducing and extending the values given in Tables 1-5. Not only did he locate several errors, but he made a number of good suggestions which improved the presentation of the method. Also, I have taken the liberty of borrowing some of his values to fill out Table 1.

Department of Mathematics

University of California, Berkeley

Berkeley, California 94720

1. B. Baillaud \& H. Bourget, Correspondance d'Hermite et de Stieltjes, Tome 1, GauthierVillars, Paris, 1905.

2. W. E. BRIGGS \& S. ChOWLA, "The power series coefficients of $\varsigma(s)$," Amer. Math. Monthly, v. 62,1955, pp. $323-325$.

3. J. J. Y. LiANG \& John TodD, "The Stieltjes constants," J. Res. Nat. Bur. Standards Sect. $B$, v. 76B, 1972, pp. 161-178.

4. J. L. W. V. Jensen, "Sur la fonction $\varsigma(s)$ de Riemann," Comptes Rendus, v. 104, 1887, pp. 1156-1159.

5. J. P. GRAM, "Note sur le calcul de la fonction $\varsigma(s)$ de Riemann," K. Danske Vidensk. Selskab Forhandlingar, 1895, pp. 305-308.

6. B. C. BERNDT, "Chapter 8 of Ramanujan's second notebook," J. Reine Angew. Math., v. 338, 1983, pp. 1-55.

7. T. M. APOSTOL, "Formulas for higher derivatives of the Riemann zeta function," Math. Comp., v. 44, 1985, pp. 223-232.

8. R. SitARAmaChANDRARAO, "Maclaurin coefficients of the Riemann zeta function," $a b$ stracts Amer. Moth. Soc., v. 7, 1986, p. 280.

9. M. Abramowitz \& I. A. Stegun, Editors, Handbook of Mathematical Functions, Nat. Bur. Standards, Washington, D.C., 1964.

10. R. Lienard, Tables Fondamentales à 50 Décimales des Sommes $S_{n}, u_{n}, \Sigma_{n}$, Centre de Documentation Universitaire, Paris, 1948. 